\title{
LA JURISPRUDENCIA DE LA «CRISIS» DEL TRIBUNAL CONSTITUCIONAL PORTUGUÉS ${ }^{1}$
}

\author{
MIGUEL NOGUEIRA DE BRITO² \\ Profesor de Derecho Público \\ Universidad de Lisboa
}

SUMARIO

I. Introducción. II. La jurisprudencia de la «crisis», de 2011 a 2014. III. Una crítica de la jurisprudencia. IV. La jurisprudencia de la crisis como afirmación de soberanía.

\section{INTRODUCCIÓN}

La jurisprudencia del Tribunal Constitucional sobre las medidas de austeridad dictadas en los últimos tiempos, y recogidas principalmente en las leyes presupuestarias para los años 2011 a 2015, ha suscitado un importante debate doctrinal y una extensa literatura sobre el tema ${ }^{3}$. En estas notas expondremos dicha jurisprudencia, incidiendo de forma particular en los parámetros utilizados por el Tribunal Constitucional para invalidar algunas de las medidas de austeridad adoptadas; con esa base trataremos de abordar una cuestión ciertamente relevante, así, en qué medida dichas decisiones del Tribunal Constitucional pueden

1 Traducción de Fernando Reviriego Picón, profesor Titular de Derecho Constitucional de la UNED.

2 Ex Letrado del Tribunal Constitucional.

3 Cf. Blanco de Morais, C., Curso de Direito Constitucional - Teoria da Constituição em Tempo de Crise do Estado Social, Tomo II, Vol. 2, Coimbra Editora, 2014, pp. 709 y ss.; De Almeida Ribeiro, G., Pereira Coutinho, L. (dirs.), O Tribunal Constitucional e a Crise: Ensaios Críticos, Almedina, Coimbra, 2014; ReIs Novais, J., Em Defesa do Tribunal Constitucional: Resposta aos Críticos, Almedina, Coimbra, 2014. Vid. también Fasone, C., Constitutional Courts Facing the Euro Crisis. Italy, Portugal and Spain in a Comparative Perspective, EUI Working Paper MWP 2014/25, disponible en http://cadmus.eui.eu/bitstream/handle/1814/33859/ MWP_WP_2014_25.pdf; Nogueira DE Brito, M., «Putting social rights in brackets? The Portuguese experience with welfare rights challenges in times of crisis», European Journal of Social Law, n. ${ }^{\circ}$ 1-2, 2014, pp. 87-102. 
ser entendidas o vistas como una expresión de afirmación de la soberanía del Estado.

\section{LA JURISPRUDENCIA DE LA «CRISIS», DE 2011 A 2014}

Podemos considerar que se encuentra ya concluido el primer ciclo de la jurisprudencia de la crisis (esperemos que sea el único), esto es, el ciclo que acompaña la aplicación del Programa de Asistencia Económica y Financiera (PAEF) en el período que media entre 2011 y 2014.

Como es bien sabido este programa vino originado por la petición de ayuda financiera que Portugal solicitó al Fondo Monetario Internacional, la Comisión Europea y el Banco Central Europeo en mayo de 2011, y tras la que se firmó un Memorándum de Entendimiento sobre las Condiciones de Política Económica y con el que Portugal obtuvo un préstamo de setenta y ocho mil millones de euros ${ }^{4}$.

Puede afirmarse, grosso modo, que la política de austeridad instaurada por el Gobierno, durante el período de vigencia del PAEF, se estructuró en tres grandes bloques: (i) reducción de los salarios de los empleados del sector público, incluyendo a quienes reciben salarios abonados por entidades públicas, desde los titulares de los órganos que representan la soberanía popular a trabajadores de las empresas públicas, e incluyendo recortes similares en las pensiones, (ii) medidas de austeridad sobre beneficiarios de los diferentes regímenes de seguridad social, (iii) flexibilización del despido de trabajadores en las funciones públicas.

Ello sin tener en cuenta, claro está, el terrible aumento de la presión fiscal sobre todos los contribuyentes, con independencia de que los rendimientos provinieran del sector público o del sector privado.

Una presión fiscal que, por cierto, se fue incrementando al tiempo que el Tribunal Constitucional fue invalidando algunas de las medidas de austeridad adoptadas, concretamente las relacionadas con la primera de las dimensiones aludidas (recortes en salarios y pensiones). Basta mencionar, a título de ejemplo, cómo Portugal fue el país de la OCDE donde más se incrementó la carga fiscal en 2013 respecto a $2012^{5}$. De hecho, según el Instituto Nacional de Estadística, la presión fiscal aumentó en 2013 un 8,1\%, después de una disminución observada en 2012, y que se corresponde aproximadamente con el 34,9\% del PIB (32,4\% en el año anterior). Este aumento de la carga fiscal se debió en gran medida al crecimiento de los impuestos directos $(25,7 \%)$, al tiempo que

4 Disponible en versión portuguesa, en http://www.portugal.gov.pt/media/371372/mou_pt_2011 0517.pdf.

5 http://economico.sapo.pt/noticias/portugal-lidera-aumento-da-carga-fiscal-na-ocde-em-2013_207877. html (consultada el 29 de marzo de 2016). 
se mantenían los impuestos indirectos y las contribuciones sociales experimentaban un aumento de $2,3 \%{ }^{6}$.

\section{Las reducciones salariales de los funcionarios y la igualdad proporcional}

Tal vez, las medidas de austeridad más significativas, y de las que el Gobierno esperaba que tuvieran un mayor impacto dentro del esfuerzo de consolidación fiscal, fueron las reducciones en los salarios de los trabajadores en el sector público introducidas por los Presupuestos del Estado para 2011, 2012 y 2013, y sobre las que el Tribunal Constitucional se pronunció a través de las Sentencias 396/2011, 353/2012 y 187/2013.

\section{A) El Presupuesto del Estado para 2011 y la Sentencia 396/2011}

El artículo 19, apartados 1 y 2 de la Ley 55-A/2010, de 31 de diciembre (Ley de Presupuesto para 2011) redujo las retribuciones de los trabajadores públicos entre un $3,5 \%$ y un $10 \%$, dependiendo del importe las mismas, a un amplio abanico de personas, identificadas en el apartado 9 del mismo artículo, así desde el Presidente de la República hasta los trabajadores de las empresas públicas, pasando por la propia judicatura. No se incluyeron en la relación, cuestión significativa, a los funcionarios del Banco de Portugal.

Quedaron afectados todos aquellos que gozaban de salarios mensuales abonados por el erario público, superiores a mil quinientos euros, incluidos los representantes de la soberanía, demás órganos constitucionales y cargos públicos, militares de las Fuerzas Armadas y de la Guardia Nacional Republicana, gerentes públicos y similares, y los trabajadores de la administración central, regional y local del Estado, así como las empresas, fundaciones e instituciones públicas. A través de los artículos 20 y 21 de la misma ley se introdujeron similares reducciones en las remuneraciones para los jueces y fiscales.

Con relación a esta ley, la Sentencia 396/2011 comenzó negando la existencia de un derecho fundamental a la no reducción de la remuneración, teniendo en cuenta que «una cosa es el derecho a la retribución, y otra bien distinta el derecho a una cuantía concreta, y no reducible por ley, con independencia de las circunstancias y de las variables económicas y financieras que la puedan condicionar». Ahora bien, en tanto que la irreductibilidad salarial no sería «una dimensión garantista recogida en el ámbito de protección del derecho a la retribución por el trabajo», y no planteándose «la afectación del derecho a un salario mínimo labo-

$6 \mathrm{http} / / / \mathrm{www}$. ine.pt/xportal/xmain? xpid=INE\&xpgid=ine_destaques\&DESTAQUESdest_ boui $=211100098 \&$ DESTAQUESmodo=2 (consultada el 29 de marzo de 2016). 
ral, en tanto que la reducción de la remuneración sólo afecta a salarios superiores a mil quinientos euros, valor muy superior a aquel, dicha irreductibilidad sólo podría provenir del principio de protección de la confianza y buena fe y del principio de igualdad».

Por lo que hace referencia al principio de protección de la confianza, el Tribunal consideró que no puede razonablemente ponerse en duda que «las medidas de reducción de la remuneración tienen por objeto salvaguardar el interés público que debe considerarse prevalente y ello constituye una razón decisiva para rechazar la alegación de que nos encontramos ante una falta de protección contraria a la Constitución». Y agregó: «A la situación de desequilibrio presupuestario y a la apreciación que ello suscitó en las instancias y en los mercados financieros internacionales se atribuyen generalmente riesgos ciertos de sacudir los cimientos (o incluso el colapso) del sistema económico y financiero nacional, lo que también se materializaría en graves consecuencias para la calidad de vida de los ciudadanos. Las reducciones salariales forman parte de un conjunto de medidas que el poder político, de común acuerdo con las organizaciones internacionales de las que Portugal es miembro, se tomaron para reequilibrar las cuentas públicas, teniéndose por absolutamente necesarias para evitar consecuencias desastrosas en la esfera económica y social. Se trata de medidas de política financiera, esencialmente coyunturales, para combatir una situación de emergencia, por la que optó el poder legislativo debidamente legitimada por el principio democrático de representación popular».

Por último, en lo referente al principio de igualdad, el Tribunal afirmó no querer inmiscuirse en el debate político y económico sobre la mayor o menor bondad de las medidas implementadas, correspondiéndole únicamente «evaluar si las soluciones impugnadas son arbitrarias, haciendo recaer excesivas cargas en un sector específico de los ciudadanos».

En este sentido, leemos en la Sentencia 396/2011 que «No podemos afirmar que ese sea el caso (que se trate de una decisión arbitraria). El hecho de no prescindir de una reducción de salarios, en el marco de distintas medidas articuladas de equilibrio presupuestario, que también incluyen subidas de impuestos y otros recortes del gasto público, se basa en una racionalidad coherente con una estrategia de actuación cuya definición todavía se encuentra dentro del margen de libre configuración política del legislador. Se intenta así lograr a corto plazo —obligados por compromisos suscritos con organismos europeos e internacionales-, resultados en el lado del gasto, donde sólo la reducción de los salarios garantiza cierta eficacia inmediata, siendo por ello una medida indispensable. No habiendo evidencias en sentido contrario, y dentro de lo que cabe calificar como «límites de sacrificio», de que la temporalidad y los montantes de la reducción la fugacidad y las reducciones todavía salvaguardan, debe aceptarse que nos encontramos ante un medio no sólo legítimo sino necesario, dentro del contexto actual, para reducir el gasto público, con el fin de re-equilibrar el presupuesto. A la vista de esta finalidad, el sacrificio adicional que se le exige a quien cobra del erario públi- 
co — vinculado, es oportuno recordarlo, a la búsqueda del interés público— no constituye un tratamiento desigual que pueda calificarse de injustificado».

B) El Presupuesto del Estado para 2012 y la sentencia 353/2012

En el presupuesto del Estado de 2012, el legislador trató de introducir nuevas medidas para limitar los salarios de los trabajadores en el sector público, añadidas a las ya establecidas en el Presupuesto de 2011 y que serían prorrogadas. Así, el artículo 21.1 de la Ley 64-B/2011 de 30 de diciembre, estableció que durante la vigencia del Programa de Asistencia Económica y Financiera se suspendieran, como medida excepcional de estabilidad presupuestaria, las pagas de vacaciones y Navidad o cualesquiera otras prestaciones correspondientes a las pagas extras, a los empleados del sector público cuyo salario básico mensual fue superior a mil cien euros. A su vez, conforme al apartado 2 del mismo artículo, los trabajadores del sector público con sueldos entre seiscientos y mil cien euros quedarían sujetos a una reducción proporcional de las mismas prestaciones.

El artículo 25 de la misma Ley estableció medidas similares para las personas que recibiesen pensiones de jubilación a través del sistema público de seguridad social.

Estas medidas fueron consideradas inconstitucionales en virtud de la Sentencia 353/2012 del Tribunal Constitucional, sobre la base de la violación del principio de igualdad.

Fue en esta decisión donde el Tribunal desarrolló el principio de igualdad proporcional; considerando las reducciones para los trabajadores con ingresos superiores a mil cien euros (reducciones de aproximadamente el 14,3\% del ingreso anual respectivo), el Tribunal fue sensible al argumento de que «ninguno de los sacrificios descritos tienen equivalentes para la mayoría del resto de ciudadanos que reciben ingresos de otras fuentes, independientemente de sus cantidades».

Por lo tanto, según el Tribunal, «la diferencia de trato resulta tan acentuada y significativa que las razones de eficacia de la medida adoptada en su persecución del objetivo de reducir el déficit público a los valores indicados en los memorandos de entendimiento, carecen de relevancia suficiente para justificar una diferencia de tal tamaño, tanto más cuando existe el recurso a soluciones alternativas para la reducción del déficit, bien por el lado del gasto (por ejemplo, las medidas contenidas en los memorandos de entendimiento), bien por el de los ingresos (por ejemplo, a través de medidas integrales_de efecto equivalente a la reducción de los ingresos). Tales soluciones pueden llegar a ser lo suficientemente eficientes desde el punto de vista de la realización del interés público, en orden a permitir aliviar la situación de los otros contribuyentes que reciben salarios o prestaciones sociales pagadas por fondos públicos». En consecuencia, para el Tribunal resultaría «evidente que la diferencia de trato impuesta a quien recibe remuneraciones o 
pensiones de los fondos públicos vulnera el principio de proporcionalidad con relación al derecho a la igualdad».

A la par que se declararon inconstitucionales las normas contenidas en los artículos 21 y 25 de la Ley de Presupuestos Generales del Estado para el año 2012, el Tribunal reconoce que resulta esencial para el Estado portugués, en el actual contexto de situaciones de emergencia, continuar teniendo acceso a la financiación que resultaba del PAEF, de modo que «el cumplimiento de tal valor presupuestario se revela, por tanto, de excepcional interés público».

En este contexto, el Tribunal sostuvo que «encontrándose en ejecución avanzada los presupuestos de 2012, debe reconocerse que las consecuencias de la declaración de inconstitucionalidad, podrían determinar de forma inevitable poner en peligro la obtención de adecuada financiación y en consecuencia la solvencia del Estado».

El Tribunal decidió así, al amparo de lo previsto en el art. 282.4 del texto constitucional, restringir los efectos de la declaración de inconstitucionalidad, dejando de lado la regla general de la eficacia ex tunc de sus decisiones. No obstante, la restricción de los efectos significó aquí, de forma inédita, que los mismos no se producirían, no solo desde la entrada en vigor de las leyes hasta el momento de la decisión de la Corte, sino que también se extenderían más allá y hasta el final de 2012. De este modo, no quedó afectada la supresión de las pagas de vacaciones y navidad o prestaciones equivalentes durante 2012.

\section{C) El Presupuesto del Estado para 2013 y la Sentencia 187/2013}

El legislador, intentando adecuarse a las disposiciones de la Sentencia 353/2012, redujo las medidas de austeridad en la Ley de Presupuestos para el año 2013; así lo hizo mediante el artículo 29 de la Ley 66-B/201, de 31 de diciembre. Además del mantenimiento de la reducción de remuneraciones introducida por la Ley de Presupuestos de 2011, se determinó, durante la vigencia del Programa de Asistencia Económica y Financiera, como medida excepcional de estabilidad presupuestaria, la suspensión del pago de la retribución de las vacaciones o de los otros beneficios correspondientes a las pagas extras para los empleados del sector público cuyo salario mensual básico fuera superior a 1100 euros.

A semejanza de lo acontecido en la Sentencia 396/2011, el Tribunal Constitucional, en esta posterior Sentencia 187/2013, entiende que esta nueva medida de reducción de salario no suponía una violación del derecho a la retribución o al principio de protección de la confianza. Pero, también en la línea de lo previsto en la Sentencia 353/2012, entiende que se produce una violación del principio de igualdad proporcional.

En esta ocasión, el Tribunal consideró que, a la vista de la medida descrita, «una intervención con un alcance reducido (sólo) de la remuneración de los asalariados de las Administraciones Públicas no resulta, dado lo anterior, en sí mis- 
ma arbitraria». La Corte incluso reconoció que «otras opciones de base, como política de consolidación fiscal, resultan teóricamente admisibles (aparte de la reducción de los gastos de remuneración de los empleados en las funciones públicas), pero también afirmó que «precisamente esta capacidad de definir las líneas de actuación política, sujeta a controversia y debate en las instancias correspondientes, está reservado al legislador democráticamente legitimado». Pero, según el Tribunal, la «conclusión de que en la reducción salarial prevista en el artículo 29 de la Ley 66-B/2012 subyace un criterio de ponderación racionalmente sostenible no resulta, no obstante, suficiente para asegurar su respectiva validez constitucional».

Es en este contexto donde se retoma la idea de igualdad proporcional como parámetro de control de constitucionalidad de la medida restrictiva contenida en el Presupuesto del Estado para 2013. Según el Tribunal «la igualdad proporcional implica la consideración del grado de diferenciación impuesto, siquiera su relación con las finalidades perseguidas — que presupone que las medidas diferenciadoras se impongan en el grado necesario, adecuado y no excesiva desde el punto de vista del interés que se pretende proteger (cfr. las sentencias 634/1993 y 187/2001)_, siquiera dentro del ámbito de comparación que se hace entre los sujetos afectados por la medida y los que no y, desde el punto de vista de esa finalidad, entre unos y otros con el Estado. Respecto de los límites del sacrificio impuesto a estos sujetos: después de un cierto punto, este aumento del sacrificio se traduce en un trato injusto y desproporcionado, no pudiendo justificarse por las ventajas comparativas que la consolidación fiscal puede presentar comparado con otras alternativas disponibles». De esta forma, según el Tribunal, en la línea de sus decisiones precedentes, señala que «los efectos acumulativos y continuados de los sacrificios impuestos a las personas con salarios en el sector público, sin equivalencia para la generalidad de otros ciudadanos que reciben ingresos de otras fuentes, implica una diferencia de trato que no encuentra justificación suficiente en el objetivo de reducción del déficit público. E implica por ello una violación del principio de proporcionalidad, basado en la idea de que la desigualdad justificada por la diferencia de situaciones no es inmune a un juicio de proporcionalidad y no puede revelarse excesivo».

\section{D) El presupuesto del Estado para 2014 y las Sentencias 413/2014 y 574/2014}

En la Ley de Presupuestos para 2014, el legislador intentó concentrar las medidas de restricciones salariales. En vez de conjugar los recortes salariales del $3,5 \%$ al $10 \%$ de los salarios mensuales superiores a 1100 euros con la suspensión de las prestaciones correspondientes a las vacaciones, como ya hizo en los Presupuestos de 2012 y 2013, la intención del legislador ahora durante 2014 fue contener las remuneraciones brutas mensuales superiores a seiscientos setenta y cinco euros obtenidos por los trabajadores del sector público con reducciones de $2,5 \%$ 
al $12 \%$ siendo este último porcentaje el que grava los ingresos de dos mil euros, según lo establecido en el artículo 33 de la Ley 83-C/2013, de 31 de diciembre.

Apreciando la constitucionalidad de la norma, el Tribunal Constitucional consideró que ello correspondía «a un acentuación significativa en el grado de diferenciación» entre los trabajadores del sector público y el sector privado. De esta forma, también la medida de reducción de remuneración contenida en la Ley de Presupuestos Generales del Estado para el 2014 se consideró contraria al principio de proporcionalidad.

Como consecuencia de la Sentencia 413/2014, la Asamblea de la República aprobó un proyecto de ley gubernamental que, esencialmente, pretendía combinar «(a) la reducción de la remuneración aplicable a 2014 a los trabajadores pagados con fondos públicos equivalente a las vigentes hasta 2013, con (b) una reducción de la remuneración equivalente al $80 \%$ de esta, en 2015, y con (c) un programa normativo, orientado a la eliminación de los recortes salariales que vienen afectando a los trabajadores, en los tres próximos años», como puede leerse en la Sentencia. 574/2014, dictada, en control previo y que estimó la conformidad constitucional del proyecto analizado.

Con relación a 2014, el Tribunal Constitucional en su Sentencia 574/2014 consideró que se justificaba el mantenimiento de la reducción remuneratoria, en conexión con lo decidido en la Sentencia 413/2014. En cuanto a 2015, se afirmó en la citada Sentencia 574/2014 que «si por una parte, supone una vuelta a la normalidad o al menos a un nivel sin el mismo nivel de restricciones de opciones presupuestarias que vino marcando los años 2011-2014, no es menos cierto que la pendencia de un procedimiento por déficit excesivo, tras un período de asistencia económica y financiera, también dibuja un cuadro particularmente exigente de excepcionalidad, capaz de restar la imposición de reducciones de salarios este año a la censura del principio de igualdad. Destaca, a su juicio, los términos más mitigados del sacrificio impuesto, por efecto de la instauración de reducciones en la remuneración inferiores en un 20\% a las previstas para 2014». Por último, en lo que respecta al período posterior, la misma sentencia consideró que el propósito de condicionar la reversión de la medida de recorte salarial «a la reducción cuantitativa de la masa salarial, obtenida a partir de la disminución en el número de funcionarios públicos» no justificaba, a la luz del principio de igualdad, que «la compensación de los empleados pagados con fondos públicos, y sólo éstos, continúen afectados por las reducciones durante esos tres años». Para el Tribunal Constitucional, y teniendo en cuenta la exigencia de igualdad en la asignación del gasto público, «no es constitucionalmente admisible la estrategia de reequilibrio de las finanzas públicas basadas en la reducción del gasto a través de la continuidad del sacrificio de esos mismos trabajadores». A este respecto, el Tribunal declaró inconstitucionales las disposiciones del artículo 4 del Decreto 264/XII de la Asamblea de la República, que preveían que en los «presupuestos del Estado para 2016 y en los presupuestos posteriores, se establezca el porcentaje de reversión de la reducción en la remuneración en función de la disponibilidad presupuestaria». 
Tras este fallo del Tribunal se aprobó la Ley 75/2014, de 12 de septiembre, que determinó la aplicación con carácter transitorio de las reducciones salariales que habían sido admitidas por aquel durante la vigencia de la PAEF y que definió los principios que deberá cumplir la correspondiente reversión, a partir de 2015.

Y así, después de este largo brazo de bierro, concluyeron las medidas de reducción salarial adoptadas por el Gobierno en virtud de la política de austeridad.

\section{Las medidas relativas a las pensiones de los regímenes de la Seguridad Social y de la Caja General de Depósitos}

Una segunda dimensión relevante dentro de la jurisprudencia de la crisis es aquella que incide en la cuestión de los recortes en materia de pensiones. Aquí podemos hacer referencia a tres decisiones del Tribunal Constitucional: (i) en primer lugar, los fallos sobre la contribución extraordinaria de solidaridad contenida en las sentencias 187/2013 y 472/2014; (ii) en segundo lugar, la decisión sobre la convergencia de las pensiones, contenida en la Sentencia 862/2013 (esto es, la convergencia entre las pensiones de los funcionarios inscritos en la Caja General de Depósitos y todos los trabajadores inscritos en la Seguridad Social); y (iii) por último, la decisión relativa a la contribución de sostenibilidad, aplicable a todas las pensiones pagadas por un sistema público de protección social contenida en la Sentencia 575/2014.

\section{A) La contribución extraordinaria de solidaridad (CES)}

El artículo 78 de la Ley 66-B/ 2012, de 31 de diciembre estableció una medida con la misma nomenclatura de lo ya previsto por las leyes de presupuestos para 2011 y 2012 (artículos 162.1 de la Ley 55-a/2010, de 31 de diciembre, y 20.1 de la Ley 64-B/2011, de 30 de diciembre), aunque se caracterizó, genéricamente, no sólo por el aumento de su base imponible, sino tambien por la ampliación del espectro de las pensiones afectadas. De hecho, en la Ley de Presupuestos Generales del Estado para 2013 llegaron «a estar sujetas a la contribución extraordinaria de solidaridad pensiones de montante significativamente inferiores (a partir de mil trescientos cincuenta euros, afectando la medida, además, a las pensiones pagadas por las entidades pública, a todas las prestaciones pecuniarias vitalicias debidas por cualquiera que fuera el título correspondiente a jubilados, en la reserva, prejubilados o figuras equivalentes que no están expresamente excluidos por ley», como afirmó en la Sentencia 187/2013, que se pronunció sobre este cuerpo normativo.

Conforme esta misma sentencia, «son irrelevantes para este fin, por fuerza de ley, la denominación de prestaciones (pensiones, subvenciones, subsidios, rentas, seguros, indemnizaciones por cese de actividad, prestaciones concedidas en el 
ámbito de los fondos de pensiones colectivos o de otro tipo), la forma que adopten (por ejemplo, pensiones complementarias, regímenes profesionales), bien de naturaleza pública, privada, cooperativa u otra, y el grado de independencia o autonomía de la entidad (incluyendo las soportadas por instituciones públicas, entidades reguladores, de supervisión o control, empresas públicas, de ámbito nacional, regional o municipal, planes de pensiones profesionales y por personas colectivas de derecho privado o cooperativo».

Las tarifas de la CES establecidas en el artículo 78 varían progresivamente desde el 3,5\% de todas aquellas pensiones mensuales que oscilen entre mil trescientos cincuenta y mil ochocientos euros, hasta el $40 \%$ del montante que supere dieciocho veces el valor de la base de cotización (es decir, la cantidad de siete mil quinientos cuarenta y cinco euros).

El CES fue cuestionado con base en diferentes argumentos: en primer lugar, por afectar a los regímenes de pensiones complementarias, incluso de fuentes privadas, por lo que resultaría dudosa su conformidad con la tutela constitucional de la propiedad privada; en segundo lugar, admitida su calificación como un impuesto, debería regirse en ese caso por los principios de unidad y universalidad en la Constitución; en tercer lugar, se puso también en duda la conformidad de la CES con los principios de igualdad, proporcionalidad y protección de la confianza.

El Tribunal Constitucional comenzó por considerar que la aportación analizada «incluso cuando resulta exigida a los titulares de los complementos de pensiones está, por ello, estrechamente asociada a los efectos de la seguridad social, y que estos pensionistas se benefician de la solvencia del sistema y aprovechan las ventajas de su financiación, en la medida en que el fortalecimiento de la protección social que se puede obtener por la vía de la asignación de prestaciones complementarias, sólo se torna efectivo si el Estado continúa satisfaciendo puntualmente las prestaciones concedidas por el sistema de bienestar, de las que también son destinatarios».

Con relación a la violación del derecho de propiedad, en virtud de la reducción de la cantidad de prestaciones que se deben en materia de pensiones, la misma «apenas podría sostenerse si fuese posible afirmar un estricto principio de correspondencia dentro del ámbito de la relación jurídica de seguridad social, de manera que existiera equivalencia efectiva entre el montante de las cuotas y el valor de las prestaciones». El Tribunal reconoció, sin embargo, que «el argumento no tiene la misma validez en cuanto a los complementos de pensiones, que funcionen conforme a un esquema de capitalización. Sin embargo, como se ha señalado, los regímenes complementarios están vinculados al sistema de seguridad social en su conjunto, y analizándose la incidencia de una contribución similar a las cotizaciones de los trabajadores en activo, no se alcanza a ver en qué condiciones dichos rendimientos deben encontrarse cubiertos por el alcance de la protección del derecho de propiedad, cuando todavía estamos en el campo de la parafiscalidad». 
Con relación a la cuestión de su caracterización como impuesto, el Tribunal consideró que «tratándose de una contribución a la seguridad social a pesar de su carácter atípico, no queda sujeto a los principios tributarios generales, y en particular los principios de unidad y universalidad del impuesto, no siendo aplicables al caso las reglas del artículo 104.1 de la Constitución relativas al impuesto sobre los ingresos de los empleados». Lo que permitió al Tribunal evitar la clasificación de la CES como impuesto, fue su encuadramiento «como tertium genus de otras contribuciones financieras a los servicios públicos», a que pasó a hacer referencia, junto a los impuestos y tasas, en la línea de lo previsto por el artículo 165.1 de la Constitución».

Finalmente, con relación a los principios de igualdad, proporcionalidad y protección de la confianza, parece haber sido decisivo en el examen realizado por el Tribunal, la circunstancia de que la CES fue «concebida exclusivamente para satisfacer, junto con otras medidas, la situación de crisis económica y financiera, también de forma temporal, dentro del abanico de opciones tomadas por el poder político, un refuerzo urgente de la financiación del sistema de seguridad social a expensas de los propios beneficiarios». Según el Tribunal, «atendiendo a la naturaleza excepcional y temporal de la medida, con la finalidad de satisfacer los objetivos de déficit público requeridos por el Programa de Asistencia Económica y Financiera, su conformidad con los principios estructurales del Estado democrático de derecho debe ser avalada».

Así, concretamente, respecto del principio de protección de la confianza, el Tribunal considera que «a la vista de la limitación que rodeaba el desarrollo de la contribución extraordinaria de solidaridad, no sólo las expectativas de estabilidad en el sistema legal parecen más moderadas, sin que existen razones relevantes de interés público que justifican, ponderadamente, una excepcional y transitoria interrupción del comportamiento de los Estados». Del mismo modo, el carácter excepcional y temporal del CES justificó también en última instancia su admisibilidad a la luz del principio de proporcionalidad.

Por su parte, el artículo 2 de la Ley 13/2014, de 14 de marzo, introdujo cambios que agravaron el régimen de la CES conforme el artículo 76 de la LOE de 2014, afectando a las pensiones de cuantía superior a mil euros. Con relación a estas alteraciones la Sentencia 572/2014 observó por primera vez, alabando las declaraciones contenidas en la exposición de motivos del proyecto de ley que dio lugar a la Ley 13/2014, de 14 de marzo, y la nota explicativa del Gobierno, que «el CES asume, efectivamente, un carácter excepcional y transitorio, directamente relacionado con los objetivos inmediatos de equilibrio presupuestario y la sostenibilidad de las finanzas públicas que el legislador afirma que quiere continuar».

Asegurada así la naturaleza transitoria o temporal de la CES, ahora en su cuarto año de vigencia, el Tribunal Constitucional consideró que se trataba de un «factor que debe ser tenido en cuenta en la subyacente ponderación del juicio de constitucionalidad». 
Así fue, de hecho. En cuanto al principio de protección de la confianza, se planteó al Tribunal Constitucional determinar «si la réplica de la medida en la LOE de 2014, con una extensión de la respectiva base de reserva y la modificación de las tasas efectivas aplicadas en alguno de los escalones legalmente definidos, sigue encontrándose dentro de los límites establecidos por los principios jurídicos constitucionales, tal y como han venido siendo diseñados por la jurisprudencia reiterada y constante de este Tribunal». Después de varias consideraciones, en donde domina la afirmación del carácter excepcional y transitorio de la medida, acaba por concluir en la citada Sentencia 572/2014 que «no parece que la reedición de la CES en la LOE de 2014, con los cambios ahora establecidos, justifique, en la perspectiva específica del principio de protección de la confianza, una evaluación sustancialmente diferente de la que recayó sobre la análoga medida análoga de la LOE de 2013». Idénticas consideraciones fueron formuladas con relación al principio de proporcionalidad, llevando al tribunal a «aceptar que la prestación mensual requerida a los beneficiarios de la seguridad social afectados por la ampliación de la base de reserva del CES, por la temporalidad de las normas que le dan soporte y los objetivos perseguidos, no constituye un sacrificio particularmente excesivo e irrazonable».

B) La convergencia de pensiones entre los regímenes de la Seguridad Social y de la Caja General de Depósitos

Sobre una materia un tanto diversa debió pronunciarse el Tribunal Constitucional en la Sentencia 862/2013, en sede de control previo, por la inconstitucionalidad del art. 7 del Decreto 187/XII, que estableció mecanismos convergencia del sistema de protección social de la función pública (Caja General de Depósitos) con el régimen general de la seguridad social. En este contexto, el referido art. 7 afectó a los pagos de pensiones de la Caja General de Depósitos, disminuyendo su valor respectivo.

Según el Tribunal Constitucional la afectación del pago de las pensiones implicaría una violación de las expectativas de los respectivos beneficiarios, que «sólo encontraría justificación en el contexto de una reforma estructural que integrase de forma amplia la ponderación de varios factores. Sólo una reforma de este tipo podría justificar eventualmente un cambio en las cuantías de las pensiones a pagar, debiendo ir acompañada de otras medidas que procediese a realizar reequilibrios en otras áreas. Una medida que podría aplicarse con el fin de reducir la cantidad de pago de la pensión tendría que ser aquella que encontrase un fuerte apoyo dentro de una solución sistémica, estructural, diseñada de manera efectiva para lograr los tres objetivos antes establecidos: sostenibilidad del sistema público de pensiones, igualdad proporcional, y solidaridad intergeneracional». Dado que no este sería el supuesto concreto, se entiende que la disposición analizada violó, a criterio del Tribunal, el principio de protección de la confianza. 


\section{C) Una contribución de sostenibilidad (CS)}

A la reforma estructural reclamada por el Tribunal Constitucional para justificar una alteración de las cuantías relativas a las pensiones abonadas por la Caja General de Depósitos respondió el legislador con la creación de la contribución de la sostenibilidad, contenida en el Decreto 262/XII de la Asamblea de la República. No obstante, sometida a control preventivo fue declarada inconstitucional por la Sentencia 575/2014.

Conforme se afirma en la referida Sentencia 575/2014, «la reducción definitiva de la cuantía en el importe de las pensiones en curso de pago se justifica en la exposición de motivos que acompaña al proyecto de ley presentado a la Asamblea de la República, precisamente por las exigencias derivadas del «contrato intergeneracional». Y lo es por tres razones: en primer lugar, porque, según se dice, el contrato no se cumplirá si la disciplina presupuestaria a la que está obligado el Estado portugués no está satisfecha; en segundo lugar, porque, según se dice, el contrato no se cumplirá si la cuestión de la sostenibilidad del sistema de seguridad social no se resuelve en la actualidad; en tercer lugar, debido a que, el contrato no se cumplirá en sus términos si la generación actualmente beneficiaria del sistema (los pensionistas actuales) no contribuyen a la financiación del sistema. Por tanto, se fundamenta que una reducción definitiva de las pensiones en curso de pago sea considerada por el legislador como una contribución de sostenibilidad».

El Tribunal, como se indica en la misma resolución, «no puede dejar de reconocer el relevante peso que, a la luz de la Constitución, revisten cada uno de esos fundamentos. Si la consistencia de los derechos afectados se encuentra acentuada, en los parámetros aplicables, no podrá ser menor la exigencia de la necesidad de su afectación, dada la relevancia de los derechos o intereses, también los constitucionalmente protegidos, que la justifican, conforme la exposición de motivos presentada a la Asamblea de la República. Este punto resulta crucial, una vez que el método de ponderación, anteriormente explicado, no puede ser aplicado con rigor si no toma en cuenta el peso específico que posee cada uno de los elementos a valorar. Siendo intenso el grado de insatisfacción de un principio constitucional (en este caso, el principio de protección de las expectativas legítimas de los pensionistas para recibir un beneficio definido y adquirido al abrigo de la normativa anterior), más intensa será la razón que justifique esa falta de satisfacción. Ello significa que la asignación de derechos de los pensionistas sólo puede no tenerse en cuenta, en este caso ya a la luz de la Constitución, si se considera necesaria para cumplir con «los derechos e intereses constitucionalmente protegidos que debe considerar prevalentes».

Sin embargo, según el Tribunal, «el interés de la sostenibilidad del sistema público de pensiones, realizado a través de una simple medida de reducción de la cuantía del importe de la pensión, sin tener en cuenta otros factores que pudieran ser relevantes para mitigar la lesión de posiciones jurídicas de los pensionistas especialmente en el plano de igualdad y equidad interna y de la justicia intrage- 
neracional e intergeneracional- y falto también de una justificación suficiente que pueda disipar las dudas sobre la idoneidad y necesidad de la medida, no puede considerarse como un interés público superior frente a la intensidad del sacrificio que se impone a los particulares.

\section{Cesación de la relación de empleo público y otras modificaciones en la legislación laboral}

Por último, la Sentencia 474/2013 declaró, en el curso de un proceso de control previo, la inconstitucionalidad de las normas contenida en el Decreto $177 / \mathrm{XII}$, que permitía el cese del contrato de trabajo en funciones públicas transcurrido el plazo de doce meses tras la ubicación del trabajador en situación de expectativa de destino y sin que se produjera el reinicio de las funciones, y ampliando los supuestos de ubicación en situación de recalificación. Las mismas reglas todavía permitían el régimen descrito a los trabajadores del sector público con vinculación permanente, sin sujeción al régimen del contrato de trabajo que había sido salvaguardado por la Ley de 2008.

A través de la citada Sentencia 474/2013, el Tribunal Constitucional consideró que la posibilidad de la resolución del contrato de trabajo tras la ubicación en situación de recalificación viola la garantía constitucional de seguridad en el empleo y el principio de proporcionalidad, prevista en los artículos 53 y 18, párrafo 2, de la Constitución; por otro lado, se entiende también que la sujeción a ese mismo régimen de los trabajadores con nombramiento definitivo violó el principio de protección de la confianza ${ }^{7}$.

\section{UNA CRÍTICA DE LA JURISPRUDENCIA}

Una vez descrita, en sus elementos principales, la jurisprudencia de la crisis, corresponde ahora reflexionar críticamente sobre la misma.

\section{La elección de los parámetros: principios generales en lugar de los derechos fundamentales}

El primer aspecto que debemos tener en cuenta es que el Tribunal Constitucional adoptó como parámetros constitucionales, a la luz de los que acabó final-

7 En este sentido, también hay que mencionar el resto de decisiones del Tribunal Constitucional: la Sentencia 602/2013, sobre la eliminación de un cierto número de días festivos obligatorios y el mecanismo de mejora de los días del período anual de vacaciones, o la Sentencia 794/2013 sobre el aumento del horario normal de trabajo en las funciones públicas. 
mente de adoptar decisiones de inconstitucionalidad, no los derechos sociales de los trabajadores, como el derecho a la retribución ${ }^{8}$ o el derecho a la seguridad social, al menos de forma uniforme, sino principios constitucionales generales, tales como el principio de la igualdad, el principio de protección de la confianza y el principio de proporcionalidad.

Esta opción continuó con tal coherencia que vale la pena mencionar los diversos casos en que la misma se manifiesta.

\section{A) Derecho a la retribución}

El Tribunal decidió que el derecho a la retribución no es, al menos con relación a su respectivo valor, objeto de protección constitucional.

Sobre este derecho, afirmó en su Sentencia 396/2011 que «no se recoge en la Constitución regla alguna que establezca de forma directa y autónoma, una garantía de irreductibilidad de los salarios». Como señaló de forma destacada en la misma resolución, «derecho fundamental, ese sí, es el «derecho a la retribución», y derecho de naturaleza análoga a los derechos de libertad y garantías, como es doctrina pacífica del Tribunal (véase, por ejemplo, la Sentencia 620/2007). Pero una cosa es el derecho a la retribución, y otra bien diferente el derecho a una cuantía específica, irreductible por la ley, cualesquiera que sean las circunstancias y las variables económicas y financieras que de forma concreta lo condicionan. No puede por tanto entenderse que la imposibilidad de afectación salarial sea una dimensión garantista contenida en el ámbito de protección del derecho a la retribución del trabajo o que una reducción de la cuantía en la remuneración se traduzca en una afectación o limitación de este derecho».

Esta misma idea fue reafirmada en la Sentencia 187/2013, concluyéndose también allí que «no se aplica el estatuto iusfundamental al derecho a la irreductibilidad de la prestación, ni como derecho autónomo, materialmente constitucional, ni como una dimensión primaria del derecho fundamental a la justa retribución consagrada en el artículo 59.1.a de la Constitución».

\section{B) Derecho a la pensión}

Del mismo modo, en cuanto a la disposición incluida en la Ley de Presupuestos Generales del Estado para 2013 que suspende el pago de vacaciones de jubilados, en la reserva, la Sentencia 187/2013 consideró que «el reconocimiento

8 Respecto al mismo, se reafirma en la Sentencia 187/2013, el «entendimiento, expresado en la Sentencia 396/2011, de que no se aplica el estatuto iusfundamental al derecho la irreductibilidad de la prestación, ni como derecho autónomo, materialmente constitucional, ni como una dimensión primaria del derecho fundamental a una retribución justa consagrada en el art. 59.1.a) de la Constitución». 
de los derechos de pensión y a la protección específica de que gozan no impide, en principio, la posibilidad de reducir el montante concreto de la pensión. Lo que está constitucionalmente garantizado es el derecho a una pensión, no a una cantidad concreta, en concepto de pensión. Esto es consecuencia de la aplicación de los criterios legalmente establecidos, pero de valor infraconstitucional», se considera asimismo que el derecho en cuestión no goza de la protección constitucional de la propiedad privada.

En otros supuestos, sin embargo, el criterio del Tribunal parece más cuestionable.

\section{C) Los derechos de pensión en virtud de regímenes complementarios}

En primer lugar, no se comprende la Sentencia 187/2013 en lo relativo al CES, al menos en la medida en que la misma empezó a afectar a las prestaciones abonadas al abrigo del régimen complementario de la iniciativa colectiva. Como es bien sabido este régimen abarca, de conformidad con el artículo 81.2 de la Ley de Bases de la Seguridad Social (Ley 4/2007, de 16 de enero, modificada por la Ley $83-\mathrm{A} / 2013$, de 30 de diciembre) un régimen público de capitalización y sistemas complementarios de iniciativa colectiva y de iniciativa individual. Según el Tribunal «los regímenes complementarios están vinculados al sistema de seguridad social en su conjunto, y abordándose la incidencia de una contribución similar a las cotizaciones de los trabajadores en activo, no se ve en qué términos dichos ingresos deben encontrarse cubiertos por el alcance de protección del derecho de propiedad, cuando todavía estamos en el campo de la parafiscalidad». Esta argumentación es claramente insuficiente, no aclarando exactamente lo que se quiere mostrar con la afirmación de que «todavía estamos en régimen de parafiscalidad». Si eso está claro, por lo que respecta a las prestaciones pecuniarias abonadas por la Seguridad Social en el marco del sistema de bienestar, cuando se analizan prestaciones sustitutivas de los rendimientos del trabajo, perdidas a consecuencia de la verificación de las eventualidades legalmente definidos (tales como el desempleo, invalidez o vejez) ${ }^{9}$, deja de estarlo en relación con las prestaciones con arreglo al plan complementario. En este último caso, las prestaciones se asientan en un régimen de capitalización, al contrario de lo que ocurre con el sistema de bienestar, basado en un sistema de solidaridad. De hecho, los regímenes complementarios, que de hecho son de adhesión voluntaria, lo que acontece es la garantía de una protección social complementaria que surge como contrapartida de prestaciones realizados por los respectivos beneficiarios.

Según el Tribunal, los regímenes complementarios se encuentran «especialmente regulados en los artículos 81 y siguientes de la Ley 4/2007, y también en

9 Cf. Vasques, S., Manual de Direito Fiscal, reimp., Almedina, Coimbra, 2015, pp. 264 y ss.

UNED. Teoría y Realidad Constitucional, núm. 38, 2016, pp. 575-602 
los de iniciativa privada que forman parte del sistema de seguridad social, con base en el principio de complementariedad, traducido en la articulación de las diversas formas de protección social pública, social, cooperativa, mutualista y privada con el fin de mejorar la cobertura de las situaciones y promover el reparto de responsabilidades». Queda por esclarecer en qué medida los participantes en dichos regímenes se benefician de un «reparto de responsabilidades», o incluso lo que se entiende por este concepto.

La Ley no hace ninguna referencia al respecto.

\section{D) Derecho a la asistencia material en caso de desempleo involuntario}

En segundo lugar, la Sentencia 187/2013 se pronunció sobre el artículo 117 de la Ley de Presupuestos para 2013, que sometió los importes de las subvenciones concedidas en virtud de las contingencias de enfermedad o desempleo, a una «contribución», respectivamente, de un $5 \%$ y un $6 \%$. Esta contribución sería deducida por las instituciones de seguridad social de la cantidad de prestaciones pagadas por ellas constituyendo un ingreso del sistema de bienestar. En ambos casos se trata de prestaciones contributivas (incluídas en el régimen general de la seguridad social contributiva y obligatoria) y sustitutorias del rendimiento del trabajo (sistema de bienestar).

Según el Tribunal, de los artículos 59 y 63 de la Constitución se deriva un derecho de los trabajadores a la «asistencia material cuando involuntariamente se encuentren en situación de desempleo y dirige un mandato al legislador para proveerlo en el sistema de seguridad social, con formas de asistencia material a los trabajadores en situación de enfermedad. Este fue el imperativo que el legislador procedió a ejecutar en el marco subsistema de pensiones, a través de los regímenes jurídicos de protección social en las eventualidades de desempleo y enfermedad».

Sin embargo, para el Tribunal, la Constitución no garantiza el derecho a una cantidad específica de asistencia material, incluso en caso de desempleo, por lo que la irreductibilidad en el montante de las prestaciones no está incluida en el ámbito de protección del derecho de los trabajadores a la asistencia material en situación de desempleo y, a fortiori, en el contexto del derecho a la asistencia en la situación de enfermedad. Únicamente no sería de este modo si la reducción en cuestión fuera tal que llegara a descaracterizar estas prestaciones, convirtiendo en inviable la función de protección de la salud por ellas desempeñada (sustitución de la remuneración) y dándoles un cariz que poco las diferenciaría de las prestaciones asistenciales. Pero no sería el caso de una reducción de un 5 o $6 \%$ como la aquí analizada. Sin embargo, de acuerdo con la Corte «a pesar de que no puede ser puesto en duda la reversibilidad de los derechos concretos y las expectativas creadas subjetivamente con base en ellos, no puede dejar de reconocer que siempre habrá de quedar a salvo que, en caso de emergencia económica, el núcleo de 
la existencia mínima ya ejecutada por la ley general que regula el derecho a las prestaciones en los casos de enfermedad o desempleo, pone también en juego el parámetro constitucional de existencia digna».

A pesar de este razonamiento, el Tribunal llegó a la conclusión, en términos que no resultan claros, de la violación del principio de proporcionalidad, contenido en el artículo 2 del texto constitucional. De hecho, si el contenido esencial de la existencia mínima se vio afectada, ¿Por qué razón no concluir la violación del derecho social a la asistencia material en situación involuntaria de desempleo?

Lo anteriormente apuntado se vuelve todavía más evidente si consideramos que en la Sentencia 413/2014 el Tribunal consideró inconstitucional una norma similar a la que se acaba de mencionar. La nueva norma incluía una cláusula de salvaguardia, con el fin de evitar que la aplicación de la contribución sobre prestaciones de enfermedad y desempleo, pudiera perjudicar, en cualquier caso, la «garantía de un valor mínimo de prestaciones en los términos previstos en los respectivos sistemas».

El Tribunal sostuvo que, incluso con esta novedad no se impidió la infracción del principio de proporcionalidad, ya que en la Sentencia 187/2013 había apuntado «también la atención en la falta de razonabilidad de la medida cuando llega a los beneficiarios que se encuentran en situación de mayor vulnerabilidad, por no disponer de las condiciones para obtener los rendimientos del trabajo, para satisfacer las necesidades básicas de su hogar, y cubre los beneficios sociales que son, precisamente, la función de sucedáneo de remuneración salarial de la que el empleado fue privado».

Ahora, según el Tribunal, «este otro fundamento parece tener plena acogida independientemente de la superación de la garantía del valor mínimo de las prestaciones en el punto donde la Corte asocia la falta de razonabilidad a la especial vulnerabilidad de los destinatarios de la medida debido a los beneficios atribuidas, lo que implica que ya, por su propio régimen, una disminución pecuniaria sustancial en relación a los normales rendimientos de trabajo, y no tanto el mayor debilitamiento resultante de esas prestaciones, por efecto de la contribución, por debajo del nivel mínimo concretado normativamente». Para ello, la Corte recurre a la construcción doctrinal de Jorge Reis Novais que apunta un cuarto requisito en la evaluación de proporcionalidad ${ }^{10}$. Una vez más, e incluso con mayor motivo, no se llega a comprender por qué motivo no admite el Tribunal un derecho social a la asistencia material en caso de desempleo involuntario.

Cabe cuestionarse así, como apunta Gomes Canotilho, en qué medida la línea jurisprudencial seguida por el Tribunal Constitucional no equivale a «poner entre paréntesis los propios derechos económicos, sociales y cultura-

10 Cf. Reis Novais, J., As Restrições aos Direitos Fundamentais Não Expressamente Autorizadas pela Constituição, 2. ${ }^{a}$ ed., Coimbra Editora, 2010, pp. 765 y ss.; idem, Os Princípios Constitucionais Estruturantes da República Portuguesa, Coimbra Editora, 2004, pp. 187 y ss. 
les» y transformarlos en una «metodología funcional de la obtención de salario decisorio» ${ }^{11}$.

\section{La reconceptualización del principio de igualdad}

En lugar de encuadrar las cuestiones constitucionales suscitadas en el contexto de los derechos sociales, la Corte optó por adoptar como parámetro de constitucionalidad de las medidas de austeridad los principios de igualdad, proporcionalidad y protección de la confianza. La opción de la Corte parece cuestionable si consideramos la forma en la que fue desarrollada a lo largo de la jurisprudencia de la crisis. Esta opción implicó, con relación al principio de igualdad, una reformulación del mismo, incluyendo, en particular, la delimitación del correspondiente campo de aplicación, y una profundización del razonamiento de la Corte en cuanto a su aplicación, en particular, en la coherencia con su jurisprudencia previa en la materia. Ya en lo atinente a los principios de protección de la confianza y de proporcionalidad, lo que estaba esencialmente en causa era precisamente la cuestión de la coherencia con la anterior jurisprudencia.

En la Sentencia 353/2012 la Corte invalidó la medida de suspensión de los subsidios de vacaciones de Navidad y de los trabajadores del sector público con base en la violación de la igualdad proporcional. A pesar de algunos antecedentes en la utilización de esta fórmula, la misma supone, sin duda, una modificación de la doctrina jurisprudencial sobre el principio de igualdad.

El Tribunal fue más allá de la igualdad y la prohibición de la discriminación arbitraria, de hecho, incluso reconoció que esta prueba podría ser satisfecha por las medidas analizadas. Así, en la Sentencia 187/2013 se afirmó que «una intervención con un alcance reductor (exclusivamente) de la remuneración de los asalariados de las Administraciones Púbicas no resulta, en vista de lo anterior, en sí misma arbitraria».

Resulta así necesario aclarar (i) lo que la Corte entiende por igualdad proporcional y (ii) los casos a que resulta aplicable este test de igualdad, supuestamente más exigente que la igualdad como prohibición de discriminación arbitraria. Y aquí comienzan las dificultades.

La igualdad proporcional puede significar, en primer lugar, la introducción del principio de proporcionalidad en la estructura del control de igualdad. Parece ser algo similar a lo que que el Tribunal tiene en mente cuando afirma, en la Sentencia $187 / 2013$ que «la diferencia de trato de los trabajadores del sector público no puede ser justificada por el carácter más eficaz de las medidas de reducción de salarios, en detrimento de otras alternativas posibles de contención de costes». El problema

11 Cf., en este sentido Gomes Canotilho, J.J., «"Bypass” social e o núcleo essencial das prestações sociais», Estudos sobre Direitos Fundamentais, Coimbra Editora, Coimbra, 2008, p. 244. 
estriba aquí obviamente, en conocer las posibles alternativas que habría que valorar a la luz del subprincipio de necesidad, integrado en el de igualdad.

$Y$ téngase en cuenta que el problema resulta tanto mayor en tanto que no resulta convincente la argumentación desarrollada por la Corte para aceptar la mayor eficacia de las medidas analizadas como razón justificadora de la diferencia de trato en relación con los trabajadores del sector privado, en detrimento de otras posibles razones. Estas otras razones consistían en una mayor seguridad de la relación laboral de los trabajadores del sector público con relación a los trabajadores del sector privado y en el mayor nivel retributivo, de media, de los trabajadores en el sector público frente al sector privado. Conforme al parecer de la Corte dichas razones no serían justificables:

«No dejan de tener aquí relevancia las consideraciones antes expuestas, que no permiten dar por sentada la idea de la inmutabilidad de la relación laboral del empleo público - ahora sujeta para la mayoría de los trabajadores que desempeñan funciones públicas a un régimen similar a lo previsto para la relación laboral privada - y también la idea, ya expresada en la citada Sentencia 353/2012, de no resultar posible establecer un cuadro comparativo, a nivel cuantitativo, de las remuneraciones recibidas en cualquiera de los sectores de actividad, por estar implicados diferentes tipos de trabajo y regímenes profesionales.

Con independencia de la dificultad de medir, en términos cualitativos, la situación general de los trabajadores del sector público en comparación con la de los trabajadores del sector privado, lo cierto es que desde el punto de vista de la distribución del gasto público, lo que es comparable es la posición subjetiva de un trabajador de la Administración Pública con un trabajador con una relación laboral privada, con empleo a tiempo completo, y la misma capacidad de ingreso, o con cualquier otro titular de un ingreso de idéntica entidad.

La imposición de sacrificios más intensos para los trabajadores que ejercen funciones públicas no puede justificarse por factores macroeconómicos relacionados con la recesión económica y el aumento del desempleo, que deben ser solucionados por medidas de política económica y financiera de carácter general, y no a través de una mayor penalización o castigo de los trabajadores que, en el plano de la empleabilidad, no soportan, o no en el mismo grado, los efectos efectos recesivos de la situación económica»(§ 43).

Esta argumentación no se revela suficiente y llevaría antes a considerar que, si no es posible «establecer un cuadro comparativo, a nivel cuantitativo, de las remuneraciones recibida en cualquiera de los sectores de actividad», y es difícil de medir «en términos cualitativos, la situación global de los trabajadores del sector público en comparación con la de los trabajadores del sector privado», entonces habría sido preferible no haber establecido ninguna comparación entre los regímenes de trabajo del sector público y el privado y tratar de sacar las consecuencias de esta comparación, en el plano de la violación del principio de la igualdad.

En lugar de ello, la Corte optó, de forma artificial, y en sentido contrario a su anterior jurisprudencia, donde siempre hizo hincapié en la necesidad de no hacer 
comparaciones parciales de las situaciones jurídicas, por considerar «comparable la posición subjetiva de un empleado de la Administración Pública con la de un trabajador con una relación laboral privada, con pleno empleo y misma capacidad de ingreso, o con cualquier otro titular de rendimientos de magnitud similar».

A esta argumentación cabe oponer, por supuesto, el juicio formulado por el Tribunal en su Sentencia 683/99: «Intentar abstraerse de todo eso, para analizar apenas un aspecto del régimen, para calificarlo como desventajoso y fulminarlo por ser inconstitucional por la violación del principio a la igualdad, no resulta aceptable» ${ }^{12}$.

La complejidad de la argumentación del Tribunal resulta todavía mayor si tenemos en cuenta que la misma Sentencia 187/2013 consideró inconstitucional la norma que impone una reducción en la remuneración de la paga extraordinaria de los trabajadores del sector público (artículo 45 de la Ley de Presupuestos para 2013). Ahora, según el Tribunal, a pesar de existir una diferencia en las cantidades percibidas por este concepto, entre el sector público y el sector privado, «esta diferencia no se da en un valor tal que permita identificar, sin duda alguna, una situación razonable de la desigualdad. Esto se debe a que otros elementos de la situación del empleo, relevante en el mismo campo de valoración y, por lo tanto, a tener que ser considerado en una ponderación más amplia — pensamos de forma especial en el horario normal de trabajo-, distinguen de forma clara los dos sectores, con un favorecimiento global del trabajo en la función pública» (cf. § 52). Si en efecto, el carácter más favorable (en relación con el sector privado) de las horas normales de trabajo en el servicio público justifica una reducción de las horas extraordinarias en ese contexto, ¿por qué razón no iba el régimen más favorable de la cesación del vínculo laboral en la función pública (con relación a la misma cuestión en el sector privado) a justificar una reducción salarial para los trabajadores que se benefician de ella?

Del mismo modo, la justificación de las reducciones de remuneración en los trabajadores del sector público con base en una mayor estabilidad del empleo público, aunque rechazada como una razón que justifique la diferencia de trato respecto de los trabajadores del sector privado, sin embargo, se admitió para intensificar el marco generador de confianza en el mantenimiento de esa estabilidad en la Sentencia 474/2013 (cf. el punto 15.1 de este último) ${ }^{13}$.

12 El contexto en que esta afirmación se realizó fue el de la duda sobre «la legitimidad de una comparación parcial, es decir, de un determinado aspecto del régimen jurídico de los contratos de trabajo celebrados entre particulares y de los constitutivos de la relación jurídica de empleo público». Lo que se analizaba era la conversión en contratos de trabajo indefinido dentro del sector público y en el ámbito de la ley general del trabajo.

13 En la Sentencia 413/2014, el Tribunal validó también una disposición de la Ley de Presupuestos para 2014, que admitió la reducción, y hasta supresión, del beneficio social del cónyuge o compañero de un contribuyente del sistema de seguridad social en caso de muerte del asegurado (la llamada «pensión de supervivencia»). De acuerdo con esta disposición, la reducción podría tener lugar en caso de que el beneficiario ya estuviese cobrando de otros beneficios sociales. Una vez más, el Tribunal afirmó que esta disposición no suponía una violación del derecho constitucional a la seguridad social. Sin embargo, el Tribunal consideró que la 
La Corte también podría haber entendido la igualdad proporcional como exigencia de un equilibrio entre las razones de la diferenciación y la medida de esa misma diferenciación. También en este caso las consideraciones anteriores conservan toda su razón de ser.

Si la conceptualización y aplicación de la igualdad proporcional puede resultar problemática en los términos expuestos, hay otro aspecto en el que suscita dificultades. Así, la cuestión de conocer como resolverá en el futuro el Tribunal Constitucional la delimitación entre esta fórmula de igualdad y la fórmula tradicional de la prohibición de discriminación arbitraria. Hasta ahora había una respuesta sencilla a las diferencias en la intensidad del control jurisdiccional en materia de igualdad: si estuvieran en juego categorías dudosas como las incluidas en el artículo 13.2 del texto constitucional la Constitución, o derechos fundamentales, se justificaba un control más intenso. El futuro, en cuanto al alcance del ámbito de aplicación de la igualdad proporcional, corresponde al Tribunal Constitucional, sin que tengamos todavía posibilidad de predecir la dirección a la que orientará su jurisprudencia aquel ${ }^{14}$.

\section{La doble vertiente de la protección de la confianza}

Las cuestiones que, a nuestro modo de ver, se sitúan en relación con los demás parámetros de control invocados por el Tribunal no se anclan tanto como una construcción jurídica ensayada por el Tribunal, sino con la consistencia del modo en que esos principios fueron aplicados.

El problema de la coherencia resulta, de hecho, exacerbado por la doble vertiente de la protección de confianza. Conforme la jurisprudencia del Tribunal Constitucional, la invocación de una violación del principio de confianza exige «en primer lugar, que el Estado (especialmente el legislador) haya iniciado comportamientos capaces de generar en los particulares expectativas de continuidad; en segundo lugar, dichas expectativas deben ser legítimas, justificadas o fundadas en buenos motivos; en tercer lugar, los particulares deben tener hechos planes de vida esperando una continuidad del comportamiento estatal; por último, es nece-

prestación violaba el principio de igualdad, una vez que afectaba especialmente a los beneficiarios de la "pensión de supervivencia» afectados por oposición a los beneficiarios de otros beneficios sociales. La pregunta que inmediatamente surge, y a la que el Tribunal no respondió, es la de saber si esos otros beneficiarios (¿cuáles?) se encuentran en una posición comparable.

14 Sobre «igualdad proporcional», cf., también, Nogueira DE Brito, M., «Comentário ao Acórdão n. ${ }^{\circ} 353 / 2012$ do Tribunal Constitucional», Direito E Política, n. ${ }^{\circ} 1$, 2012, pp. 108-123; NoGUeIRA DE BRIto, M. Pereira Coutinho, L., «A «Igualdade Proporcional», Novo Modelo no Controlo do Princípio da Igualdade? Comentário ao Acórdão do Tribunal Constitucional n. ${ }^{\circ}$ 187/2013», Direito E Política, n. ${ }^{\circ} 4,2013$, pp. 182 y ss.; Nogueira de Brito, M., «Medida e Intensidade no Controlo da Igualdade na Jurisprudência da Crise no Tribunal Constitucional», G. De Almeida Ribeiro y L. Pereira Coutinho (orgs.), 0 Tribunal Constitucional e a Crise: Ensaios Críticos, cit., pp. 107 y ss. 
sario que no existan razones de interés público que justifiquen, una vez ponderadas, la no continuidad del comportamiento que generó la expectativa» ${ }^{15}$.

Podemos decir que las tres primeras cuestiones son «requisitos previos» ${ }^{16}$, mientras que la cuarta se presenta como la exigencia posterior, en verdad, decisiva. A través de las tres primeras, el principio de relevancia mira al pasado; a través de la cuarta, el principio lo hace el futuro. En cierta medida, podríamos afirmar que el principio puede ser representado como la cabeza de Jano, al mismo tiempo mirando hacia atrás y hacia adelante.

Como ha afirmado Maria Lúcia Amaral el principio es, en primer lugar, un método de ponderación, tal como acontece con el principio de proporcionalidad. Simplemente, «mientras que el segundo nos permite resolver las antinomias que surgen de bienes constitucionales equivalentes, y entre sí contradictorias, que coexisten en el tiempo (entre un derecho fundamental, por una parte, y la necesidad constitucional de su afectación por otro), el principio de la protección de la confianza, nos permite resolver antinomias entre bienes constitucionales equivalentes, pero donde el conflicto tiene lugar diacrónica y no sincrónicamente (entre la situación jurídica de una persona definida por el derecho antiguo, y la necesidad de alterar en el derecho nuevo, afectándolo negativamente)» ${ }^{17}$.

Tras estas consideraciones iniciales, cabe en cierta medida corregir la afirmación anteriormente apuntada sobre la decisión del Tribunal de avalar las medidas de austeridad, a la luz de los principios constitucionales generales, tales como el principio de la igualdad, el principio de protección de la confianza y el principio de proporcionalidad. De hecho, es siempre la proporcionalidad la que está en juego, especialmente en la vertiente de la ponderación entre las situaciones afectadas y los intereses públicos que justifican, o no, esta afectación.

Ahora el problema es que en el marco de esa ponderación el Tribunal Constitucional, desde la Sentencia 187/2001, entiende que el legislador tiene una

15 Esta evaluación procede de la Sentencia 128/2009. En dicha Sentencia se procedió a la sistematización de los requisitos de la anterior jurisprudencia en materia de protección de la seguridad jurídica. Donde antes se hablaba de dos presupuestos esenciales (la afectación de expectativas en un sentido desfavorable y con la que los destinatarios de las normas alteradas no pudiesen contar, como requisito anterior y, como requisito posterior, la ausencia de necesidad de salvaguardar derechos o intereses constitucionalmente protegidos que deban considerarse prevalentes) se pasó a establecer, como requisitos previos, tres exámenes, y como requisito posterior, el examen de ponderación de acuerdo a lo que se refleja en el texto. La cuestión que se plantea es la de saber si la nueva distinción de cuatro exámenes constituye «garantía de mayor seguridad o previsibilidad de contraste de normas con el principio de confianza» [cf. MотA PINTO, P., «A protección de la confianza en la «jurisprudencia de crisis», De Almeida Ribeiro, G., Pereira Coutinho, L. (coords.), O Tribunal Constitucional e a Crise: Ensaios Críticos, cit., p. 167]. Este último autor (cf. ob. cit., pp. 172-173) se pregunta, incluso, si la separación entre la cuestión de hecho de la existencia de seguridad jurídica, incluyendo eventualmente la quiebra de la seguridad (primer y tercer examen) no debería separarse de forma más nítida de la cuestión sobre la legitimidad de su protección (segundo examen). Además, se cuestiona si la distinción entre tres exámenes en el ámbito del requisito previo no sería sobretodo relevante en el ámbito de la fiscalización concreta (cf. ibidem, p. 172).

16 Cf. Mota Pinto, P., «A Proteção da Confiança na 'Jurisprudência da Crise'», cit., p. 167.

17 Cf. Amaral, M.L., «O Tempo e a Proteção da Confiança», p. 26, http://www.icjp.pt/sites/default/ files/publicacoes/files/ebook_encontrodp_final2.pdf (consultado el 30 de marzo de 2016). 
«prerrogativa de evaluación», o un «voto de confianza», al evaluar el equilibrio entre los intereses públicos perseguidos y las medidas concretas puestas en práctica (o una apreciación de la relación entre el literal de las medidas y sus efectos prácticos). Esta construcción jurisprudencial se forjó para tiempos de normalidad; el problema consiste en saber en qué medida el mismo criterio puede quedar sin efecto en tiempos de crisis. La llamada jurisprudencia de la crisis parece no haber reservado espacio a la llamada «prerrogativa de evaluacion» cuando esta probablemente se encontraría más justificada.

\section{La opción por las medidas transitorias y la negativa a los cambios estructurales}

Las consideraciones anteriores nos han llevado hasta el último punto a considerar: saber si la Corte, al tiempo que limita las medidas de austeridad, no acaba por conducir al Gobierno y al Poder legislativo a actuar solamente en un nivel excepcional y transitorio. De hecho, los cambios más importantes introducidos en el plano estructural (régimen de pensiones, cambios en la legislación laboral) fueron, todos ellos rechazados basándose en la violación del principio de confianza. Por lo tanto, la jurisprudencia de la crisis también es en gran medida una jurisprudencia del mantenimiento del status quo ex ante.

\section{La restricción de los efectos}

La jurisprudencia de la crisis ha introducido una novedad en el campo de la restricción de los efectos en las decisiones del Tribunal Constitucional, en los términos del artículo 282.4 de la Constitución. Se trata de la restricción de los efectos, operado por la Sentencia 353/2012, en los términos antes expuestos, con el objetivo de sustituir los efectos retroactivos (ex tunc), no por una producción de efectos prospectivos (ex nunc), como resulta usual, mas con unos efectos diferidos en el tiempo.

El principal problema que plantea este aplazamiento de los efectos de la decisión del tribunal se concreta en la necesaria articulación del principio de primacía de la propia declaración de inconstitucionalidad sobre la respectiva restricción de efectos. Sólo con base en tal principio resulta posible minimizar los riesgos de surgir consideraciones en la declaración de inconstitucionalidad, que presuponen una cierta devaluación de los argumentos basados en el interés público de las medidas legislativas impugnadas, que más tarde se contradicen en la limitación de los efectos ${ }^{18}$.

18 Sobre esta cuestión, cf., con más detalle, Nogueira de Brito, M., «Comentário ao Acórdão n. ${ }^{\circ}$ 353/2012 do Tribunal Constitucional», cit., pp. 120 y ss.; Guerrero Picó, C., «Portogallo», PassaGLiA, P. (ed.), Problematiche Finanziarie nella Modulazione degli Effetti nel Tempo delle Pronunce di Incostituzionalità, 


\section{La relación entre la Constitución y el Derecho de la Unión}

Por último, es importante también hacer una breve referencia a la forma en la Corte equipara la relación entre la Constitución portuguesa y el Derecho de la Unión. En esta materia es de particular interés la Sentencia 574/2014. En esta decisión, después de enunciar las diversas disposiciones del Derecho de la Unión en la materia, desde el Pacto de Estabilidad y Crecimiento al Pacto Fiscal y las reglas relativas al Pacto fiscal, objeto de transposición a la normativa interna, la Corte declaró que «la vinculatoriedad del Derecho de la Unión Europea en este campo no alcanza los medios que los Estados miembros emplean para alcanzar las metas u objetivos que se les imponen». Por otra parte, según el Tribunal, «no hay, al menos, desacuerdo entre el Derecho de la Unión Europea y el Derecho constitucional portugués. De hecho, los principios constitucionales de igualdad, proporcionalidad y protección de la confianza, que han servido como un parámetro al Tribunal Constitucional para verificar la constitucionalidad de las normas nacionales sobre materias conexas con las de los presentes autos, forman parte del núcleo duro Estado de Derecho, integrando el patrimonio jurídico común europeo al que la Unión también está vinculada».

Este enfoque permite a la Corte afirmar el carácter vinculante de las disposiciones del Derecho de la Unión y, al mismo tiempo, no atribuirles ninguna relevancia en la interpretación de las disposiciones de derecho constitucional interno. La Corte, por otra parte, establece claramente que «el hecho de admitir que las normas adoptadas y a adoptar por el legislador nacional para alcanzar los objetivos mencionados deberán cumplir con las prescripciones de la Unión Europea no tiene consecuencias desde el punto de vista de la aplicación de las normas normas constitucionales». De esta forma, a pesar de que el Tribunal reconoce la inserción del derecho nacional en un «sistema constitucional multinivel, en el cual interactúan varios sistemas normativos», en verdad deja sin sentido la interacción entre dichas órdenes.

\section{LA JURISPRUDENCIA DE LA CRISIS COMO AFIRMACIÓN DE SOBERANÍA}

Corresponde ahora analizar críticamente si el Tribunal Constitucional «actuó como último reducto de la soberanía en un Estado sujeto a control internacional» ${ }^{19}$.

Este aspecto fue resaltado por Carlos Blanco de Morais cuando apuntó que la circunstancia de residenciar en el Tribunal Constitucional la garantía de la Cons-

\footnotetext{
diciembre 2014, pp. 63 y ss., disponible en http://www.cortecostituzionale.it/documenti/convegni_seminari/ Comp191.pdf (consultado el 30 de marzo de 2016).

19 Cf., en este sentido, Blanco de Morais, C., Curso de Direito Constitucional: Teoria da Constituiçãa em Tempo de Crise do Estado Social, Tomo II, Vol. 2, Coimbra Editora, 2014, p. 774.
} 
titución en cuanto «expresión de una superior manifestación de la soberanía normativa de un Estado», transformó este órgano constitucional en el «guardián de un reducto nuclear e indisponible de la propia soberanía». El mismo autor señala que la cuestión central en debate «paradójicamente, no se encontraba ligada a los pilares medulares de la soberanía (como la política exterior, de seguridad y defensa), sino a unos derechos improbables, libertades y garantías de los trabajadores, derechos sociales de naturaleza análoga a los derechos de libertad y a los derechos sociales y a las prestaciones» ${ }^{20}$.

Esta misma idea la encontramos también en otros autores. De esta forma, Jorge Reis Novais afirma que «cuando un Tribunal Constitucional aplica y hace cumplir la Constitución de su país para invalidar leyes aprobadas por los organismos nacionales, en el plano de la ejecución de las directrices, con el apoyo y respaldo de la entidad supuestamente considerada como protectora, por lo tanto, decidiendo con efectividad, autonomía y no en un ejercicio puramente formal de la soberanía, ese país no es un protectorado».

También, de acuerdo a este mismo autor, «las pérdidas acontecidas en el ámbito de la capacidad nacional de opciones políticas imponen exigencias complementarias a la justicia constitucional. No es sólo un problema de la compensación de la pérdida de soberanía, es también una exigencia mínima de nuestra dignidad como pueblo». En este contexto, el aumento de la participación de la justicia constitucional para garantizar los derechos constitucionales tendría como objetivo compensar una «situación fáctica de pérdida real de soberanía ya verificada en el campo de la opción política democrática» ${ }^{21}$.

Junto a ello, el mismo incremento de la responsabilidad de la garantía de los derechos constitucionales por la justicia constitucional encontraría justificación «en la pérdida del alcance de la participación democrática nacional sin compensación a nivel europeo». De lo que se trata, entonces, es de garantizar los valores constitucionales «contra las imposiciones y las políticas europeas de vaciado de los derechos sociales promulgadas por el gobierno nacional $\gg^{22}$.

Por otra parte, esta misma idea también ha sido defendida por una parte de la doctrina extranjera al abordar la jurisprudencia de la crisis en Portugal. De manera destacada, Roberto Cisotta y Daniel Gallo sostienen que la decisión en la Sentencia 187/2013 equivale a una reafirmación de la soberanía social de Portugal a través del «juez de la ley y no del autor de la ley» ${ }^{23}$.

Estas consideraciones nos llevan naturalmente a plantear la cuestión de conocer (i) cómo entender este concepto de soberanía social, esto es, una soberanía

20 Cf. Blanco de Morais, C., ob. y loc. cit.

21 Cf. Reis Novais, J., Em Defesa do Tribunal Constitucional: Resposta aos Críticos, cit., p. 190.

22 Cf. Reis Novais, J., Em Defesa do Tribunal Constitucional: Resposta aos Críticos, cit., p. 191.

23 Cf. Cisotta, R., Gallo, D., 'Il Tribunale Costituzionale Portoghese, i risvolti sociale delle misuri di austerità ed il rispetto dei vincoli internazionali ed europei', 7 Diritto Umani e Diritto Internazionale 2, 2013, pp. 465 y ss. 
expresada en los derechos sociales y (ii) si esa soberanía puede ser afirmada por el «juez de la ley» contra el «autor de la ley».

La expresión «soberanía social» ha sido utilizada por varios autores sin resolver, no obstante, una ambigüedad patente.

Por un lado, la idea de la soberanía social pretende dar cuerpo a algunas exigencias de desintegración entre las cualidades de ciudadano nacional y beneficiario de las prestaciones sociales que se aprecian en la Unión Europea. La pregunta es si pueden ser beneficiarios de las prestaciones sociales ciudadanos de un Estado miembro que han sido atribuidas por otro Estado del que son residentes. Existiría así una ciudadanía social, más extensa que una ciudadanía entendida como presupuesto de ejercicio de los derechos políticos. Una soberanía social se configuraría así como un concepto más amplio que la soberanía popular o la soberanía nacional. El problema que surge aquí es, lógicamente, la relación entre estos dos conceptos, un problema que, por otro lado, no tiene una respuesta satisfactoria por parte de los autores que hablan de tal «soberanía social» ${ }^{24}$.

Desde otra perspectiva, la idea de la soberanía social, describe un proceso de reparto del poder político entre el Estado y otros modelos de gobernanza, expresión que designa los procesos de interacción y toma de decisiones llevados a cabo por los gobiernos en sentido estricto, mas también para los mercados u otras organizaciones, públicas o privadas, nacionales o internacionales, como el FMI o incluso las llamadas agencias de calificación. En pocas palabras, la soberanía dejaría de poder ser entendida como una condición propia de determinados agentes, es decir, el Estado y sus órganos, que ejercen el poder supremo sobre un pueblo en un territorio determinado, para pasar a ser vista como una estructura de relaciones que establecen los términos de un determinado dominio de existencia social $^{25}$. El objetivo sería reconocer que los mercados ejercen poder sobre una parte importante de nuestras vidas y deben, por lo tanto, estar sujetos a formas de control democrático ${ }^{26}$.

Aún no siendo posible desarrollar aquí estos conceptos, resulta claro la dificultad en la que se asienta. Por un lado, el Estado ya no se configura como el lugar exclusivo donde reside la soberanía; por otro lado, no existen formas de control democrático al márgen del Estado, aunque estas no se revelen muchas veces eficaces.

Sin perder de vista de estas dificultades, la verdad es que la respuesta a la segunda pregunta anteriormente indicada (¿puede afirmarse la soberanía por el juez de la ley contra el autor de la ley?) nos permite percibir el verdadero significado de la soberanía que aquí se aborda. La soberanía que aquí se tiene en mente

24 Cf. M. Ferrara, The Boundaries of Welfare: European Integration and the New Spatial Politics of Social Protection, Oxford University Press, 2005, pp. 125 y 11 y ss. El autor, ob. cit., p. 12, nota 1, promete una aproximación detallada al concepto de «soberanía social», pero no cumple.

25 Cf. R. Latham, «Social Sovereignty», in Theory, Culture E Society, 2000, Vol. 17, n. ${ }^{\text {4 }}$, p. 3.

26 Cf. R. LATHAM, ob. cit., p. 15. 
no es otra que la afirmación del poder del Estado frente a un poder ejecutivo y legislativo debilitado por las estructuras internacionales que representa la troika.

Lo que parece interesante es ver que esta declaración de la independencia ya no se ejercita por el poder ejecutivo o legislativo, sino por el poder judicial. Nos enfrentamos, pues, a una situación en la que la crisis de valores y principios constitucionales no es superada por el legislador, sino por el poder judicial en la cara de una supuesta crisis del propio sistema representativo. La nota importante es que contemplar en la jurisprudencia de la crisis una manifestación especial de la soberanía - es decir, una situación en la que el Tribunal Constitucional desempeña un papel especial como el último bastión de la soberanía del Estado, mucho más allá de la afirmación normal que todas las decisiones los tribunales son, por definición, las manifestaciones de soberanía— equivale a devaluar la calidad de la deliberación democrática de los actos legislativos anulados bajo esa misma ley. La situación es entonces verdaderamente grave: no sólo nos encontramos ante decisiones que invalidan los actos disconformes a la Constitución, sino con una profunda crisis política, que subyace bajo el supuesto de que las elecciones no desempeñan la función de legitimación democrática que constituye su razón de ser. En este sentido, la jurisprudencia de la crisis es, después de todo, otro acto en la tragedia de la construcción política de la Unión Europea

$$
* * *
$$

TrtLE: The crisis' jurisprudence in the Portuguese Constitutional Court

ABstract: In these notes its analyzed the crisis' jurisprudence in the Portuguese Constitutional Court, and the fact that those decisions can be seen as an expression of affirmation of state sovereignty.

Resumen: Se analiza en estas notas la jurisprudencia de la crisis del Tribunal Constitucional portugués, abordando en qué medida dichas decisiones del Tribunal Constitucional pueden ser entendidas o vistas como una expresión de afirmación de la soberanía del Estado.

KeY wORDS: Constitutional Court. Crisis. Sovereignty.

Palabras Clave: Tribunal Constitucional. Crisis. Soberanía.

FECHA DE RECEPCIÓN: 31.03.2016

FECHA DE ACEPTACIÓN: 27.07.2016 